\title{
Nonresponse bias in EC Respiratory Health Survey in Italy
}

\author{
R. de Marco*, G. Verlato*, E. Zanolin*, M. Bugiani**, J.W. Drane+
}

Nonresponse bias in EC Respiratory Health Survey in Italy. R. de Marco, G. Verlato, E. Zanolin, M. Bugiani, J.W. Drane. CERS Journals Ltd 1994.

ABSTRACT: In the three Italian centres involved in the European Community Respiratory Health Survey (ECRHS), prevalence of asthma-like symptoms was assessed through a mailback questionnaire. Since the nonresponse rate was not negligible, ranging 10-18\%, we investigated whether nonresponse bias affected the results and, if so, whether the bias could be eliminated from the final estimates of prevalence.

A screening questionnaire was sent by mail to 7,000 randomly selected subjects 20-44 yrs of age, and nonresponders were contacted again by phone. Additional information was collected on a subsample of the respondents through a clinical interview.

A logistic regression analysis showed that, except for one symptom (awakening for coughing), symptom prevalence significantly decreased from the first to the subsequent contact, when controlling for age, sex, centre and season of interview. The decrease in symptom prevalence was largely independent of smoking habits and socioeconomic status, and was seemingly caused by a symptom-related selfselection. When correcting results according to a linear regression model, observed estimates appeared to be slightly overestimated, by 4-10\%. A simulation with the Italian data showed that the bias increased steeply at nonresponse rate higher than $30 \%$, a situation quite common in asthma surveys.

In conclusion, nonresponse bias affects the results of ECRHS in Italy, slightly inflating prevalence estimates. To make reliable comparisons on international data in the presence of different nonresponse rates, a correction of the observed prevalence seems necessary.

Eur Respir J., 1994, 7, 2139-2145.
*Cattedra di Statistica Medica e Biometria, Istituto di Igiene, Università di Verona, Policlinico di Borgo Roma, Verona, Italy. **Fisiopatologia Respiratoria, Dispensario di Igiene Sociale, Pneumologia "C", Torino, Italy. +Dept of Epidemiology and Biostatistics, School of Public Health, University of South Carolina, Columbia, SC, USA.

Correspondence: R. de Marco

Cattedra di Statistica Medica e Biometria Istituto di Igiene

Università di Verona

Policlinico di Borgo Roma

Via delle Menegone

37134 Verona

Italy

Keywords: Asthma prevalence, epidemiological surveys, mailback questionnaires, nonresponse bias

Received: February 111994

Accepted after revision September 101994

The results presented here are from a local analysis of data collected for the European Community Respiratory Health Survey. Any final international comparison may use a different form of analysis.
In epidemiological surveys on asthma, mailed and selfadministered questionnaires are the main instruments used to assess the prevalence of the disease [1]. The data available on asthma prevalence show a wide variation across different countries, which could be accounted for by several factors, such as different diagnostic criteria and/or different prevalence of risk factors. However, at least in those studies reporting final response rate, the variability in asthma prevalence (3-8\%) could be partially explained by variations in response rate (56-90\%), which appears to be negatively related to the prevalence itself [2-7].

Indeed, nonresponse can affect the estimates of the parameters, decreasing both precision and accuracy, the former being related to the number of people participating, the latter referring to the absence of bias in observed estimates. When subjects with symptoms are more likely to participate in the survey than asymptomatic subjects, as already shown in studies dealing with respiratory disorders [3], selection bias arises.

To perform reliable comparisons among different countries when response rates vary, the problem of possible selection bias must be considered. For this purpose, the process generating nonresponse has to be analysed in order to determine whether it is random [8]. When this is not the case, estimates referred exclusively to responders are biased, leading not only to incorrect conclusions about the samples under study, but also to unreliable comparisons between them [9]. The degree of bias depends upon the final nonresponse rate and the specific pattern of nonresponse [10].

When data are collected by successive contacts where the questionnaire is administered only to nonresponders to the previous contact, the process generating nonresponse can be evaluated analysing the prevalence pattern according to the contact [11-14]. Moreover, this procedure allows inference about prevalence among hard-core nonresponders, who have been reported to be more similar to late responders than to early responders [15].

The aims of this study were: 1) to analyse the nonresponse process in an international survey on asthma (European Community Respiratory Health Survey (ECRHS)) $[16,17]$ carried out in Europe, using the data of all Italian participating centres; 2) to assess the direction and the size of the bias; and 3) to evaluate the consequences of the bias in international comparisons. 


\section{Material and methods}

\section{Study design}

ECRHS is an international two-stage survey on general population aimed at testing: 1 ) whether there are significant variations in the prevalence of asthma between participating countries; and 2) whether these variations can be explained by similar variations in the prevalence of risk factors. In Italy, three centres participated in the project: Pavia, Turin and Verona.

This paper focuses mainly on the first stage of the survey, when the prevalence of some selected asthma and asthma-like symptoms was assessed through the administration of a screening questionnaire to probability samples of people 20-44 yrs of age (male/female ratio 1:1). In each centre, the random sample was drawn from an updated list of the people enrolled to the National Health Service (in Italy such enrolment is mandatory by law).

In the three Italian centres involved in ECRHS, the screening questionnaire was mailed to a sample of 1,000 people in Pavia, and 3,000 in Turin and Verona (first contact). For nonresponders to the first contact a different strategy was adopted in the three towns: in Pavia and Turin, the subjects were administered the questionnaire by phone, whilst in Verona for the second contact another letter was sent. In Verona, one more mailing and a final phone call were carried out to reach the remaining nonresponders. Phone interviews were performed by skilled personnel; interviewers were instructed to simply read the questions to the subject without adding any comments or explanations, in order to make the phone interview as similar as possible to the personal filling in of the mailing questionnaire.

The questions used in the screening questionnaire have mostly been taken from the International Union Against Tuberculosis and Lung Disease (IUATLD) Bronchial Symptoms Questionnaire (1984) developed for studies of Asthma [18]. The questionnaire, originally set up in English, was translated into Italian and the translation checked by independent back translation to English. It included seven questions on respiratory symptoms asking whether, at any time in the last 12 months, the subject: 1) had wheezing or whistling in his chest; 2) woke up with a feeling of tightness in his chest; 3) was woken up by an attack of shortness of breath; 4) was woken up by an attack of coughing; 5) had an attack of asthma; 6) was currently taking medicine for asthma; 7) suffered from any nasal allergy including "hay fever".

Information on smoking and socioeconomic status for a subsample of people attending Stage 1 came from ECRHS Stage 2. In this stage, randomly selected subjects were invited to the clinics, where, among other clinical tests [16, 17], they were administered a clinical questionnaire assessing personal and family history of respiratory diseases and relevant risk factors. The smoking habits of the subjects were classified into the following mutually exclusive groups: 1) nonsmokers - subjects who did not smoke for as long as a year, i.e. subjects who did not smoke at least 20 packs of cigarettes or 12 oz (360 grams) of tobacco in a lifetime, or at least one cigarette per day or one cigar a week for one year; 2) ex-smokers - subjects who smoked for at least one year but not in the last month; and 3) smokers - subjects who smoked for at least one year and also in the last month.

Information on employment condition was used as an index of socioeconomic status. Subjects were classified as: 1) students; 2) self-employed; 3) high-level employees (managers, foremen and supervisors working for an employer); 4) low-level employees (working for an employer, but neither a manager, supervisor or foreman); 5) not working (those people, mostly housewives, not currently employed or self-employed, and not currently looking for a job); and 6) unemployed (those people not currently employed or self-employed, and currently looking for a job).

\section{Statistical analysis}

The analysis of Stage 1 data was aimed at testing whether the symptom prevalence systematically varied throughout subsequent contacts, i.e. to check for the possible presence of selection bias, due to a correlation between readiness to respond and the presence of respiratory symptoms. For this purpose, a multiplicative logistic model was fitted to the data, using the prevalence of positive answers to each question as dependent variable, the contact order as predictive variable, and controlling for centre, season of interview, sex and age. Dates of interview were recoded into the four traditional seasons and age was split into 5 year classes and treated as a categorical variable. To make all Italian data comparable, contact was coded as a two level factor contrasting responders to the first letter versus the others. An interaction term between centre and contact was introduced into the model in order to test whether the effect of contact was constant in the three participating centres.

The selection process in Stage 2 has been studied comparing the prevalences of screening questionnaire symptoms in attenders to Stage 2 with prevalences of people refusing to attend to this stage. This analysis was feasible, since both groups of subjects did answer to the screening questionnaire in Stage 1. To assess the significance of the differences, a logistic model was fitted, controlling for centre, season of interview, age, sex and contact at Stage 1 (first vs late).

In people attending Stage 2, differences in smoking habits and employment status between early and late responders were tested with a Chi-squared test, whilst the effects of smoking and employment on screening questionnaire symptom prevalence was assessed through a multiplicative logistic model, controlling for age, sex and contact.

In order to correct observed prevalence estimates for nonresponse bias, a method proposed by DRANE [19] was used: assuming a linear trend in prevalence across subsequent contacts, the prevalence in nonresponders was extrapolated by fitting a linear regression model to the observed prevalence (Pobs) at each contact.

The discrepancy between crude (Pobs) and adjusted 
prevalence (Padj) estimates was quantitatively measured as relative difference (Bias \%):

$$
\operatorname{Bias}(\%)=100 \times((\text { Pobs })-(\text { Padj })) /(\text { Padj })
$$

Eventually the effect of nonresponse rate on the size of bias was evaluated through a simulation, in which final nonresponse rate was varied from 40 to $10 \%$, whilst keeping constant (equal to that observed) the sample size, the prevalence in early and late responders, and the ratio of response rates between the two contacts.

\section{Results}

In table 1 the sample size and the response rate at each contact are shown for each Italian centre. The final response percentage was fairly high, $86 \%$, ranging $82-90 \%$. It was higher $(\mathrm{p}<0.001)$ in females $(88 \%)$ than in males $(85 \%)$, whilst it was constant across the age groups (fig. 1). Ninety five $(10 \%)$ nonresponders explicitly refused, $106(11 \%)$ moved, $6(0.6 \%)$ died at some time during the survey, and $762(79 \%)$ could not be reached in the final contact by phone. For about $6 \%$ of the latter it was impossible to find the phone number.

Figure 2 shows the prevalence by contact and centre for tightness in chest, which exemplifies the pattern that emerged for almost all symptoms in the screening questionnaire. Prevalence decreased in late responders and, as can be seen in the Verona centre, its decrease was maximum for hard-core nonresponders. Thus, those people who did not answer three subsequent mailings and were then contacted by phone had the smallest prevalence of all groups responding.

As shown in figure 3, symptom prevalence was always higher in early than late responders. Except for breath shortness and coughing, the decrease was significant when controlling for centre, season of interview, sex and age.

Table 2 presents the percentage relative difference in prevalence estimates between early and late responders in the Italian centres. Except for coughing, the mean overestimation of prevalence in early with respect to late
Table 1. - Response rate (\%) at the successive mailings and phone contacts

\begin{tabular}{|c|c|c|c|c|c|c|}
\hline & & & Pavia & Turin & Verona & Total \\
\hline Sample & & $\mathrm{n}$ & 1000 & 3000 & 3000 & 7000 \\
\hline \multirow[t]{3}{*}{ Mailing } & I & $\%$ & 35 & 45 & 49 & 45 \\
\hline & II & $\%$ & - & - & 15 & 6 \\
\hline & III & $\%$ & - & - & 13 & 6 \\
\hline Phone & & $\%$ & 47 & 38 & 13 & 29 \\
\hline \multirow[t]{2}{*}{ Total } & & $\%$ & 82 & 83 & 90 & 86 \\
\hline & & $\mathrm{n}$ & 816 & 2502 & 2713 & 6031 \\
\hline
\end{tabular}

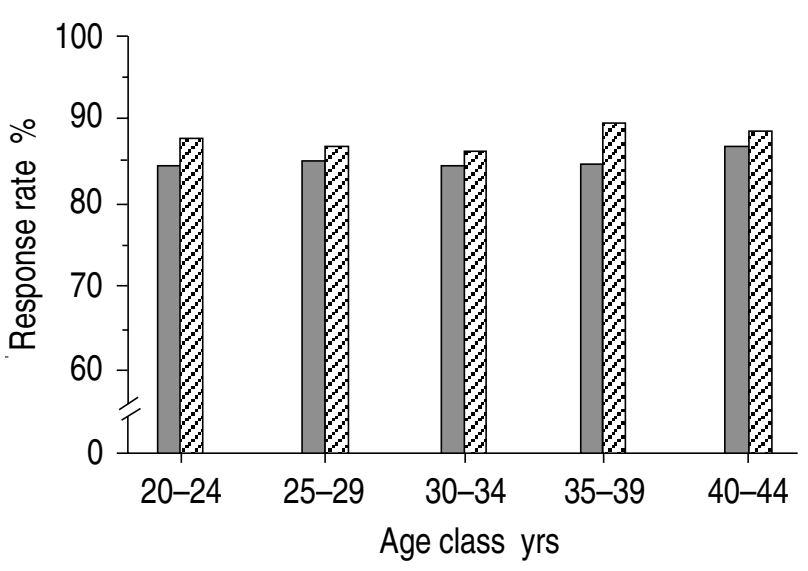

Fig. 1. - Response rates (\%) by sex and age group. $\square$ : male; $\square$ : female

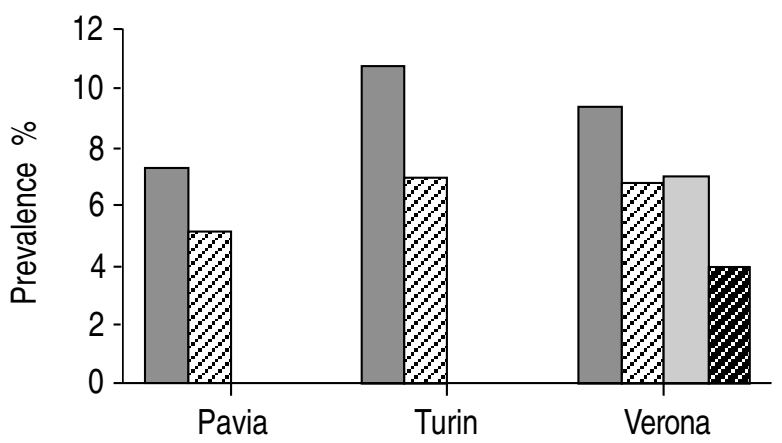

Fig. 2. - Prevalence pattern of symptom 2 (tightness in chest) as a function of contact in the three Italian centres participating in the ECRHS. $\square$ : first contact; $\square$ : second contact; $\square$ : third contact; $\square$ : fourth contact. ECRHS: European Community Respiratory Health Survey.

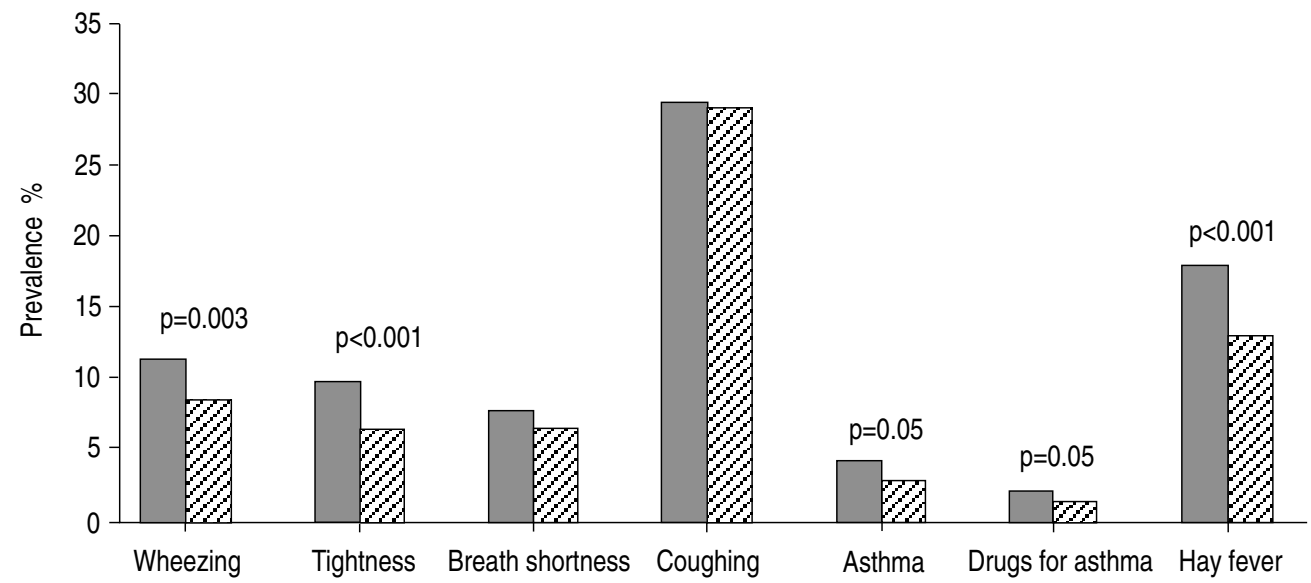

Fig. 3. - Prevalence of asthma-like symptoms in the first and subsequent contacts in the ECRHS in Italy. For each symptom, significance of differences between the first and subsequent contacts was assessed through a multiplicative logistic model, controlling for age, sex, centre and season of interview. $\square$ : first contact; $\square$ : subsequent contact(s). ECRHS: European Community Respiratory Health Survey. 
Table 2. - Relative difference in prevalence estimates (\%) between early and late responders for different symptoms in Italian centres: ((Prevalence early - prevalence late) $\div$ prevalence early) $\times 100$

\begin{tabular}{lrrcr}
\hline Symptom & Pavia & Turin & Verona & Total \\
\hline Wheezing & 35 & 34 & 33 & 34 \\
Tightness & 42 & 53 & 60 & 56 \\
Breath shortness & 40 & 17 & 19 & 21 \\
Coughing** & 0.1 & -12 & 15 & 0.1 \\
Asthma & 0 & 71 & 31 & 46 \\
Drugs for asthma & 240 & 102 & -10 & 48 \\
Hay fever* & 17 & 60 & 23 & 38 \\
\hline
\end{tabular}

Significant difference between centres assessed testing contactcentre interaction through a logistic model: *:p<0.05; **:p<0.001.

responders ranged $21-56 \%$. The degree of overestimation for almost all symptoms varied across the centres, probably reflecting different selection processes. Since the power of the test increases as a function of the number of cases, the differences among centres were significant for coughing and hay fever, the two symptoms presenting the highest prevalences, but not for asthma and drugs for asthma, the symptoms with the largest variations among centres, which, however, displayed the lowest prevalences (fig. 3).

Further information on the selection processes, which took place in ECRHS in Italy, can be obtained from the analysis of Stage 2 data. A total of 896 subjects out of a random sample of 1,829 respondents to the screening questionnaire, attended this stage, yielding an attendance rate of $49 \%$. The rate of attendance was significantly higher $(\mathrm{p}<0.001)$ for early responders to the screening questionnaire (58\%: 548 out of 945) than for late responders (39\%: 348 out of 884), indicating that the tendency to nonresponse is a "constant attitude".

In table 3 it can be appreciated that symptom prevalence, as assessed through the screening questionnaire, was remarkably greater for subjects agreeing to participate in Stage 2 than for subjects refusing. Indeed, odds ratios of attenders with respect to nonattenders, when adjusted for centre, age, sex, season of interview and contact (first

Table 3. - Prevalences of screening questionnaire symptoms in the subjects attending Stage $2(n=896)$ and in subjects selected for Stage 2 who refused to participate $(n=933)$, odds ratios (attenders versus nonattenders) and their confidence interval $(\mathrm{CI})$

\begin{tabular}{lccc}
\hline Symptoms & $\begin{array}{c}\text { Prevalence } \\
\text { attenders } \\
\%\end{array}$ & $\begin{array}{c}\text { Prevalence } \\
\text { nonattenders } \\
\%\end{array}$ & $\begin{array}{c}\text { Odds ratios* } \\
\text { (CI 95\%) }\end{array}$ \\
\hline Wheezing & 13.1 & 6.6 & $2.07(1.43-3.01)$ \\
& & & \\
Tightness & 8.5 & 5.1 & $1.87(1.22-2.87)$ \\
Breath shortness & 8.3 & 5.9 & $1.52(1.01-2.34)$ \\
Coughing & 30.8 & 26.7 & $1.22(0.85-1.55)$ \\
Asthma & 4.4 & 3.0 & $1.61(0.97-2.83)$ \\
Drugs for asthma & 2.5 & 1.4 & $1.55(0.83-3.40)$ \\
Hay fever & 18.7 & 11.5 & $1.66(1.23-2.49)$ \\
\hline
\end{tabular}

*: adjusted for centre, age, sex, season of interview and contact (first $v s$ late). $v s$ late), were always $>1$ and almost always significant, as shown when their confidence intervals do not include unity. The largest odds ratio, observed for wheezing, amounted to 2.07, indicating that the prevalence of this symptom at the screening questionnaire was almost doubled in attenders with respect to nonattenders. The odds ratios were similar in early and late responders to Stage 1, as the attendance-contact interaction term was never significant.

The analysis of Stage 2 data revealed that the nonresponse process in Stage 1, whilst seemingly unaffected by the employment status, appeared to be markedly affected by smoking habits, as reported in table 4 . Smokers tended to answer to a mail questionnaire later than exsmokers, who in turn tended to answer later than nonsmokers $(p=0.005)$. When controlling for sex, age and contact, smoking was not associated with a significant variation in screening questionnaire symptom prevalence, with wheezing as the only exception. With respect to nonsmokers, the odds ratio for wheezing was 1.8 (1.00$3.48)$ in ex-smokers and further increased up to 4.05 (2.47-6.66) in smokers. The strength of the association between smoke and wheezing was modified by contact, so that the odds ratio of smokers with respect to nonsmokers decreased from $4.82(2.72-8.56)$ in early responders to 2.70 (1.04-7.03) in late responders.

The observed prevalences at Stage 1 and the prevalences adjusted for nonresponse through the method of DRANE [19] are given in table 5 together with the bias. For most symptoms, the bias appeared to be low or moderate, ranging 4-10\%. In interpreting these values, it must be taken into account that bias has been computed considering the total response rate, which is very high, and assuming for all centres the same average decrease in prevalence between early and late responders.

The effect of different response rates on bias is shown in table 6 . It can be appreciated that bias increases rapidly as response rate decreases: when the response rate is $70 \%$ the mean bias ranges $15-21 \%$ for most relevant symptoms.

Taking into account that in comparison of asthma

Table 4. - Smoking habits and employment status ${ }^{\dagger}$ in early $(n=548)$ and late $(n=348)$ responders to Stage 1

\begin{tabular}{lrrrr}
\hline & \multicolumn{2}{c}{ Early } & \multicolumn{2}{c}{ Late } \\
& \multicolumn{2}{c}{ responders } & \multicolumn{2}{c}{ responders } \\
& $\mathrm{n}$ & $\%$ & $\mathrm{n}$ & $\%$ \\
& & & & \\
Smoking habits* & 262 & $(47.8)$ & 134 & $(38.5)$ \\
Nonsmokers & 108 & $(19.7)$ & 65 & $(18.7)$ \\
Ex-smokers & 178 & $(32.5)$ & 149 & $(42.8)$ \\
Smokers & & & & \\
Employment status & 39 & $(7.2)$ & 20 & $(5.8)$ \\
Student & 48 & $(8.9)$ & 25 & $(7.3)$ \\
Self-employed & 307 & $(56.9)$ & 197 & $(57.6)$ \\
High level employee & 84 & $(15.6)$ & 57 & $(16.7)$ \\
Low level employee & 42 & $(7.8)$ & 30 & $(8.8)$ \\
Not working & 20 & $(3.7)$ & 13 & $(3.8)$ \\
Unemployed & & & & \\
\hline
\end{tabular}

$\dagger$ : Information on smoking and employment were obtained in Stage 2. *: Chi-squared test, $\mathrm{p}=0.005$ for early and late responders. 
Table 5. - Overall observed prevalences (Pobs) at Stage 1 (\%), prevalences adjusted for nonresponse (Padj) (\%) through the method of DRANE [19], and bias (\%)

\begin{tabular}{lccc}
\hline Symptom & $\begin{array}{l}\text { Pobs } \\
(95 \% \mathrm{CI})\end{array}$ & $\begin{array}{c}\text { Padj } \\
\%(95 \% \mathrm{CI})\end{array}$ & $\begin{array}{c}\text { Bias } \\
\%\end{array}$ \\
\hline Wheezing & $9.9(9.2-10.7)$ & $9.3(8.6-10.1)$ & 6.59 \\
Tightness & $8.1(7.4-8.8)$ & $7.4(6.7-8.1)$ & 10.04 \\
Breath shortness & $7.1(6.5-7.8)$ & $6.8(6.2-7.5)$ & 4.09 \\
Coughing & $29.4(28.2-30.5)$ & $29.3(28.0-30.5)$ & 0.26 \\
Asthma & $3.7(3.3-4.2)$ & $3.5(3.0-3.9)$ & 8.50 \\
Drugs for asthma & $2.0(1.7-2.4)$ & $1.9(1.5-2.2)$ & 8.85 \\
Hay fever & $15.9(15.0-16.8)$ & $14.8(13.9-15.8)$ & 7.15 \\
\hline
\end{tabular}

P: prevalence; 95\% CI: 95\% confidence interval.

Table 6. - Adjusted prevalences (P) and bias for different response rates: results come from a simulation where response rate was progressively decreased, whilst keeping constant (and equal to the values observed in this study) sample size, prevalence in the two contacts and the ratio of response between the two contacts

\begin{tabular}{|c|c|c|c|c|c|c|c|c|}
\hline \multirow{2}{*}{$\begin{array}{c}\text { Response rate } \\
\%\end{array}$} & \multicolumn{2}{|c|}{ Wheezing } & \multicolumn{2}{|c|}{ Asthma } & \multicolumn{2}{|c|}{ Asthma drugs } & \multicolumn{2}{|c|}{ Hay fever } \\
\hline & $\mathrm{P} \%$ & Bias $\%$ & $\mathrm{P} \%$ & Bias \% & $\mathrm{P} \%$ & Bias \% & $\mathrm{P} \%$ & Bias \% \\
\hline 90 & 9.5 & 4.7 & 3.5 & 6.0 & 1.9 & 6.2 & 15.1 & 5.1 \\
\hline 70 & 8.6 & 15.4 & 3.1 & 20.4 & 1.7 & 21.4 & 13.6 & 16.9 \\
\hline 60 & 8.2 & 21.7 & 2.9 & 29.2 & 1.5 & 30.8 & 12.8 & 24.0 \\
\hline
\end{tabular}

prevalences (assuming $\mathrm{n}=3,000$ and $\mathrm{p}=0.05$ ) a relative difference of about $20 \%$ between two estimates is enough for a test to be significant, a "significant" result can be explained purely by the bias when the response rate is $70 \%$ or lower.

\section{Discussion}

Nonresponse is the rule rather than the exception in epidemiological surveys [20]. In spite of this, little attention has been paid, until now, to the methodological implications of nonresponse in comparative studies. Usually, a random process for nonresponse is assumed, so that the analysis can be limited to the observe data with the only apparent consequence of loss of precision.

Our analysis of the prevalence pattern according to the contact in ECRHS Stage 1 in Italy indicates that the nonresponse process is far from random. A self-selection operates in the sample, so that late responders, and probably also nonresponders, have a lower prevalence than early responders.

A similar and probably stronger process of selection also affects ECRHS Stage 2, where nonparticipating subjects showed a markedly reduced risk for all symptoms in the screening questionnaire (table 3).

A limitation of ECRHS Stage 1 in Italy is that, due to the previous failures of postal surveys in our country, in two centres out of three nonresponders to the first mailing were contacted by phone: so the hypothesis that the difference in prevalence can be due to difference in administration methods of the screening questionnaire cannot be ruled out. However, indirect evidence supports the hypothesis that observed differences between early and late responders are not due to different administration methods. Firstly, a Spanish study [6] on agreement between postal and phone answers to the screening questionnaire showed that different methods of questionnaire administration had no effect on the type of response. Secondly, the pattern that emerged in Italy is common to other centres where ECRHS $[6,21]$ protocol or other protocols [3] were implemented with the same method of administration at each contact. Thirdly, an analogous symptom-related self-selection was also present in ECRHS Stage 2, independent of the method of administration.

The possible role of smoking habits and socioeconomic status in nonresponse process and, as a consequence, in prevalence differences among early and late responders, has been partially investigated through the analysis of Stage 2 data. Whilst readiness to respond was not influenced by the socioeconomic status, smokers tended to respond later, in agreement with previous results [3, 22]. Since the smoking-related self-selection presented an opposite trend with respect to the symptom-related one, it could be argued that smoking habits acted as a confounder, attenuating overestimation of symptom prevalence in early responders. However, this confounding action should be of minor importance, considering that smoking was not associated with an increase in respiratory symptom prevalence, with the sole exception of wheezing, in agreement with the current literature [23]. Also, in this respect, the confounding action of smoking lost much of its strength, as the importance of smoking as a risk factor for wheezing markedly decreased from early to late responders; later responders included more smokers, who, however, presented less respiratory problems, as shown previously [24]. These results, however, must be interpreted with caution, since nonresponse in Stage 2 was remarkable, and preferentially affected late responders.

The analysis of the pattern of prevalence according to 
the contact (or attendance) both in Stage 1 and Stage 2 of ECRHS in Italy, strongly supports the hypothesis that difference in prevalence between early and late responders are almost entirely due to a symptom-related self-selection process. Nonresponse generates a particular kind of "selection bias": (early) responders are more likely to have symptoms than (late responders) nonresponders so that observed prevalences overestimate true prevalences [15]. This bias is equivalent to the "sick person effect" of case-control and clinical studies [25]. This kind of selection, both in terms of average response rates and of biased response can be specific to the particular population surveyed, which was young: 20-44 yrs of age. Older populations may be exposed to quite different selection processes, if any.

Due to the very high response rate in Italy, the bias causes a mild overestimation of prevalence estimates, ranging on average $4-10 \%$, with the sole exception of coughing. Our analysis shows that the size of the bias depends mainly upon three factors:

The nonresponse rate. The bias increases rapidly with the percentage of nonresponse. When the nonresponse rate is $30 \%$ or more, the size of the bias is enough to allow misleading conclusions in the comparison of observed data. If this factor seems not relevant for the Italian data, given the high participation in each centre, it could have a strong influence in international comparisons where response rate is expected to show great variation. The kind of symptoms. The bias affects the respiratory symptoms in the screening questionnaire to different degrees. Nonspecific and less reproducible [26] symptoms, such as coughing, are less affected. This fact agrees with the hypothesis that nonresponse implies a diseaserelated selection.

The type of selection process. This in turns can be split in two dimensions. 1) the strength; and 2) the mechanism of selection. As seen in table 2 the strength of selection is not constant throughout Italian centres but shows a great variability except for wheezing, probably reflecting different social/cultural patterns or different attitudes with respect to disease. Given a nonresponse rate, this is an independent factor which affects bias. The stronger the selection, the greater the bias. This dimension is likely to have a greater variability across European centres. The mechanism of selection is the last component which determines how the strength will influence bias. Given that the Italian design, in two out of three towns, allowed only for two points (early $v s$ late responders), a simple linear model for the selection mechanism was fitted to the data. When prevalences can be estimated on more than two contacts, other models can be chosen and tested, such as the exponential function [27], the logistic function [13] and many others [28].

As the effect of nonresponse may be different in each country and in each centre within a country, reflecting different cultural and social attitudes, the different sets of data are to be deeply analysed before drawing any decision on the opportunity to estimate prevalence using simply the observed data. The analysis should focus on the prevalence trend over time from the start of the survey, considering the subsequent contacts (order of mailing or the date of interview etc.). If such a trend emerges, observed prevalences have to be corrected in order to avoid biased estimates. Sometimes potential determinants of nonresponse (age, sex, smoking habits, socioeconomic status, etc.) are shown to explain the prevalence pattern over time. If this is the case and information on these determinants is available for the whole sample (responders and nonresponders), adjustment should be carried out by subdividing the total sample into strata according to the determinants levels, and by attributing, within each stratum, the same prevalence observed in responders to nonresponders. Otherwise, when the trend in prevalence is still present after adjusting for these confounders, or when it is not possible to know their distribution in nonresponders (as it is generally the case), the use of approximate and simple methods of correction, such as the method of DRANE [19], is desirable. When a monotonic trend in prevalence exists, this method makes it possible to obtain final estimates corrected both for known and unknown potential determinants of nonresponse. Obviously, when nonresponse is very high, no method can guarantee reliability of its estimates.

In conclusion, nonresponse rate, kind of symptoms, and type of selection process are three factors for which observed estimates have to be corrected in order to guarantee reliable international comparisons. ECRHS design, usually providing more than two consecutive contacts with nonresponders, allows the process generating nonresponse to be analysed through the data collected across the world in a more precise way than Italian data permit. This is a great advantage but requires a new view about nonresponse. This has to be considered part of the body of the information collected, and not only an irrelevant accident which leaves the observed data unchanged.

\section{Members of ECRHS-Italy}

Università di Verona: R. de Marco***, V. Lo Cascio**, C. Campello*, F. Rossi, C. Biasin, A. Cannistrà, B. Cenci, E. Destefani, M. Ferrari, M. Girotto, G. Lampronti, C. Martini, M. Olivieri, A. Poli, S. Tardivo, G. Verlato, A. Villani, M.E. Zanolin; Università di Pavia: A. Marinoni**, I. Cerveri*, R. Alesina, O. Basso, L. Berrayah, R. Brusotti, F. Fanfulla, P. Moi, M.C. Zoia; Università di Torino: C. Bucca*, C. Romano*, M. Aime, A. Cerutti, F. Chiampo, W. Gallo, G. Rola, F. Sulotto; USSL 77-Pavia: L. Casali*, C. Frati, P. Karytinos; USSL 7-Torino: M. Bugiani**, W. Arossa, E. Caria, A. Carosso, G. Castiglioni, P. Piccioni. *: principal investigators; **: local co-ordinator; ***: national co-ordinator.

\section{References}

1. Torén K, Brisman J, Jarvholm B. Asthma and asthmalike symptoms in adults assessed by questionnaires. Chest 1993; 104: 600-608.

2. Littlejohns P, Ebraihm S, Anderson R. Prevalence and diagnosis of chronic respiratory symptoms in adults. $\mathrm{Br}$ Med J 1989; 298: 1556-1560.

3. Bakke $\mathrm{P}$, Gulsvik A, Lilleng $\mathrm{P}$, Overå $\mathrm{O}$, Hanoa R, Eide GE. Postal survey on airborne occupational exposure 
and respiratory disorders in Norway: causes and consequences of nonresponse. J Epidemiol Commun Health 1990; 44: 316-320.

4. Abramson M, Kutin J, Bowes G. The prevalence of asthma in Victorian adults. Aust NZ J Med 1992; 22: 358-363.

5. Peat JK, Haby M, Spijker J, Berry G, Woolcock AJ. Prevalence of asthma in adults in Busselton, Western Australia. Br Med J 1992; 305: 1326-1329.

6. Galobardes MB, Castellsagué J, Sunyer J, Antò JM. Assessing the administration method of a screening for respiratory symptoms. Eur Respir J 1993; 6 (Suppl. 17): $485 \mathrm{~s}$.

7. Iversen M, Dahl R. Prevalence of self-reported asthma and respiratory symptoms in Denmark. EC Respiratory Health Survey Phase I. Eur Respir J 1993; 6 (Suppl. 17): 339s.

8. Rubin DB. Inference and missing data. Biometrika 1976; 63: 581-592.

9. Lindefors-Harris B-M, Eklund G, Adami H-O, Meirik O. Response bias in a case-control study: analysis utilizing comparative data concerning legal abortions from two independent Swedish studies. Am J Epidemiol 1991; 134: 1003-1008.

10. Vach W, Blettner M. Biased estimation of the odds ratio in case-control studies due to the use of ad hoc methods of correcting for missing values for confounding variables. Am J Epidemiol 1991; 134: 895-907.

11. Cochran WG. Sampling Techniques. 2nd edn. New York, Wiley, 1963.

12. Hochstim JR. A critical comparison of three strategies of collecting data from households. J Am Stat Assoc 1967; 62: 976-989.

13. Alho JM. Adjusting for nonresponse bias using logistic regression. Biometrika 1990; 77: 617-624.

14. Tennant A, Badley EM. A confidence interval approach to investigating nonresponse bias and monitoring response to postal questionnaire. J Epidemiol Commun Health 1991; 45: 81-85.

15. Scott C. Research on mail surveys. J R Stat Soc 1961; 124: 143-195.
16. Burney P, Luczynska C, Chinn S, Jarvis D. The European Community Health Survey. Eur Respir J 1994; 7: 954 960.

17. European Commission. The European Community Respiratory Health Survey. Medicine and Health, European Commission, Directorate-General XIII, Office for Official Publications, L-2920 Luxembourg.

18. Burney PGJ, Laitinen LA, Perdrizet S, et al. Validity and repeatibility of the IUATLD (1984) Bronchial Symptoms Questionnaire: an international comparison. Eur Respir J 1989; 2: 940-945.

19. Drane JW. Imputing nonresponses to mail-back questionnaires. Am J Epidemiol 1991; 134: 908-912.

20. Locker D, Wiggins R, Sittampalam Y, Patrick DL. Estimating the prevalence of disability in the community: the influence of sample design and response bias. $J$ Epidemiol Commun Health 1981; 35: 208-212.

21. Kutin J, Abramson M, Czarny D, Walters EH. Does nonresponse bias inflate estimates of asthma prevalence? Eur Respir J 1993; 6 (Suppl. 17): 486s.

22. Seltzer CC, Bosse R, Garvey AJ. Mail survey response by smoking status. Am J Epidemiol 1974; 100: 453457.

23. Sparrow D, O'Connor GT, Basner RC, Rosner B, Weiss ST. Predictors of the new onset of wheezing among middle-aged and older men. The normative aging study. Am Rev Respir Dis 1993; 147: 367-371.

24. Oakes TW, Friedman GD, Seltzer CC. Mail survey response by health status of smokers, nonsmokers, and ex-smokers. Am J Epidemiol 1973; 98: 50-55.

25. Sterling TD, Weinkam JJ, Weinkam JL. The sick person effect. J Clin Epidemiol 1990; 43: 141-151.

26. Brunekreef B, Groot B, Rijcken B, Hoek G, Steenbekkers A, de Boer A. Reproducibility of childhood respiratory symptom questions. Eur Respir J 1992; 5: 930935.

27. Drane JW, Richter D, Stoskopf C. Improved imputation of nonresponses to mailback questionnaires. Stat Med 1993; 12: 283-288.

28. Little RJA, Rubin DB. Statistical analysis with missing data. New York, John Wiley and Sons, 1987. 